\title{
L'activisme politique des descendants d'esclaves à Antananarivo : les héritages de Zoam
}

(This is an accepted manuscript version for an article published in Politique Africaine, n. 140, p. 23-40)

Cet article analyse les héritages laissés par un mouvement malgache d'inspiration marxiste, né en 1972 et composé de jeunes d'origine servile, dans les trajectoires politiques et les discours des ex-militants et des habitants des «bas quartiers » d'Antananarivo. Il considère le rôle que ce mouvement a eu dans la renégociation de la position sociale des descendants d'esclaves au cours des dernières décennies, les trajectoires de vie des militants après la disparition du mouvement et la manière dont le stigmate de l'origine servile a été reproduit, contesté et teinté de nouvelles valeurs politiques.

Dans plusieurs régions de Madagascar, comme dans d'autres contextes africains ${ }^{1}$, l'ascendance servile continue d'être fortement stigmatisée. Cet état de fait n'est que rarement devenu une référence explicite des mobilisations politiques ou un objet de débat public $^{2}$. La honte liée à l'ascendance servile s'est perpétuée malgré l'abolition officielle de l'esclavage en 1896 et a poussé la plupart des descendants d'esclaves à cacher leur origine $^{3}$. L'héritage de l'esclavage est une question qui reste difficile à aborder, qui suscite l'embarras (des descendants d'esclaves, mais aussi des descendants de personnes

1

. Voir, par exemple, M. A. Klein, « The Concept of Honour and the Persistence of Servitude in the Western Sudan », Cahiers d'Études africaines, vol. 45, n 179-180, 2005, p. 831-851 ; B. Rossi (dir.), Reconfiguring Slavery. West African Trajectories, Liverpool, Liverpool University Press, 2009; A. L. Araujo (dir.), Public Memory of Slavery: Victims and Perpetrators in the South Atlantic, Amherst, Cambria Press, 2010 ; A. Bellagamba, S. Greene et M. Klein (dir.), The Bitter Legacy. African Slavery Past and Present, Princeton, Markus Wieners Publisher, 2013.

2

. Sauf au niveau académique où, au cours des dernières décennies, plusieurs intellectuels malgaches et européens ont consacré leurs travaux à cette question. Voir, par exemple, I. Rakoto (dir.), L'Esclavage à Madagascar. Aspects historiques et résurgences contemporaines, Antananarivo, Institut de civilisations/Musée d'art et d'archéologie, 1997 ; I. Rakoto (dir.), La Route des esclaves. Système servile et traite d'esclaves dans l'Est malgache. Actes du colloque international de Toamasina (20-22 septembre 1999), L'Harmattan, Paris, 2000 ; S. J. T. M. Evers, Constructing History, Culture and Inequality. The Betsileo in the Extreme Southern Highlands of Madagascar, Leyde, Brill, coll. «African Social Studies Series », 2002 ; L. N. Razafindralambo, «Inégalité, exclusion, représentations sur les Hautes Terres centrales de Madagascar», Cahiers d'Études africaines, vol. 45, $\mathrm{n}^{\circ} 179-180$, 2005, p. 879-903; D. Regnier, «Pourquoi ne pas les épouser? L'évitement du mariage avec les descendants d'esclaves dans le Sud Betsileo (Madagascar) », Études rurales, vol. 194, 2014, p. 103-122.

3

. L'ascendance servile semble toutefois être reconnue sans honte dans certaines régions de l'île : M. L. Brown, «Reclaiming Lost Ancestors and Acknowledging Slave Descent: Insights from Madagascar », Comparative Studies in Society and History, vol. 46, n 3, 2004, p. 616-645. 
libres) et sur laquelle pèse une lourde chape de silence ${ }^{4}$. Pourtant, il s'agit d'une question fondamentale pour comprendre la structuration des champs politiques locaux. Par leur grand nombre et, donc, par la force électorale qu'ils représentent, les descendants d'esclaves ont été des acteurs importants du contexte politique postcolonial, que ce soit dans les processus de constitution de partis politiques ou dans les mobilisations populaires qui ont marqué l'histoire politique malgache. En 1972 par exemple, des groupes de jeunes descendants d'esclaves qui vivaient dans les périphéries pauvres d'Antananarivo ont trouvé dans l'activisme politique le moyen de dénoncer leur condition. Après avoir uni leurs forces à celles des étudiants en grève et des jeunes migrants originaires d'autres régions de l'île, ils ont formé un mouvement marxiste et anti-impérialiste qui a joué un rôle crucial dans la chute de la Première République et du régime de Philibert Tsiranana. Ce mouvement, connu sous les acronymes de Zoam ou Zwam (dont la multiplicité de significations sera discutée plus loin), a dénoncé et traduit en termes de classe les discriminations auxquelles les descendants d'esclaves (ou les migrants considérés comme tels, même quand ils ne l'étaient pas) faisaient face dans leur vie quotidienne. En s'appuyant explicitement sur le thème de l'esclavage, longtemps considéré comme un sujet tabou dans de nombreuses sociétés malgaches, et sur un vocabulaire marxiste, ce mouvement a élaboré un programme politique regroupant un large éventail d'acteurs sociaux aux origines, statuts et objectifs politiques très hétéroclites.

Basé sur des entretiens avec certains des ex-membres de Zoam et sur un travail de terrain effectué dans les bas quartiers d'Antananarivo (en particulier à Andavamamba et Isotry) entre 2013 et $2015^{5}$, cet article a pour objectif de retracer les héritages contradictoires que ce mouvement a laissés dans les trajectoires de vie et dans les discours des ex-militants et de ceux qui habitent aujourd'hui ces quartiers de la ville. Différents éléments seront pris en considération : le rôle que Zoam a tenu dans la renégociation de la position sociale et politique des descendants d'esclaves au cours des dernières décennies, les trajectoires de vie des militants après la disparition du mouvement et les contradictions qui peuvent naître quand le langage de classe est utilisé pour lire les discriminations entre groupes statutaires dans un contexte où statut social et conditions économiques ne se chevauchaient pas parfaitement. L'étude de l'activisme politique des militants permettra non seulement de rendre compte de la façon dont la question de l'esclavage et de son héritage a été connotée de nouvelles valeurs politiques durant la seconde moitié du siècle dernier, mais aussi d'analyser comment l'action politique a contribué à remettre en

4

. L. Freeman, «Speech, Silence, and Slave Descent in Highland Madagascar », Journal of the Royal Anthropological Institute, vol. 19, n 3, 2013, p. 600-617 ; P. M. Larson, « Reconsidering Trauma, Identity, and the African Diaspora : Enslavement and Historical Memory in Nineteenth-Century Highland Madagascar», The William and Mary Quarterly, vol. 56, n² 2, 1999, p. 335-362 ; D. Somda, «Et le réel serait passé. Le secret de l'esclavage et l'imagination de la société (Anosy, Sud de Madagascar) », thèse de doctorat d'ethnologie et de sociologie comparative, Paris, Université Paris Ouest-Nanterre, 2009.

5

. La recherche a été financée par le European Union's Seventh Framework Programme (FP7/2007-2013)/ERC Grant agreement $n^{\circ}$ 313737. Je remercie Lotte Pelckmans, Christine Hardung, Sandrine Perrot, les membres du groupe de recherche SWAB de l'Université de Milano-Bicocca ainsi que les relecteurs anonymes de Politique africaine pour leurs commentaires sur les versions précédentes de cet article. 
question les idéologies qui supportent les inégalités sociales au niveau local. L'analyse de l'impact de Zoam dans la redéfinition de la marginalisation sociale est cruciale pour comprendre comment un mouvement composé de descendants d'esclaves, même s'il n'a que partiellement atteint ses objectifs, a essayé de redonner une dignité aux groupes stigmatisés.

\section{La stigmatisation de l'ascendance servile}

Jusqu'à l'abolition officielle de l'esclavage, celui-ci structurait la plupart des sociétés de Madagascar. Les esclaves constituaient une partie numériquement importante de la population, en particulier sur les Hautes Terres de l'île où le royaume merina est devenu, entre la fin du XVIII ${ }^{\mathrm{e}}$ siècle et le début du XIX ${ }^{\mathrm{e}}$ siècle, l'un des centres de traite les plus importants de l'océan Indien ${ }^{6}$. Au cours du XIX ${ }^{\mathrm{e}}$ siècle, les rois et les reines merina ont étendu leur contrôle sur une grande partie de l'île et ont été reconnus par les puissances européennes comme souverains de l'ensemble du territoire, devenant ainsi les interlocuteurs privilégiés de l'Angleterre lors de la signature des accords de 1817 et 1820 contre le commerce des esclaves. Ne pouvant plus être officiellement vendus en dehors de l'île, les esclaves capturés pendant les guerres menées par les merina sont devenus la propriété d'un nombre relativement restreint de familles nobles (andriana) et libres (hova). Tout au long du XIX ${ }^{\mathrm{e}}$ siècle, des hommes, des femmes et des enfants ont été capturés lors d'attaques menées à l'intérieur de l'île ou amenés d'Afrique de l'Est via des canaux commerciaux qui ont perduré malgré l'abolition officielle de la traite. La mise en esclavage pouvait également être la conséquence d'un endettement ou la peine imposée pour certains crimes. Les esclaves étaient considérés comme la propriété exclusive de leurs maîtres : ils pouvaient être échangés et vendus, n'étaient pas reconnus en tant que parents légitimes de leurs enfants et étaient employés dans les travaux agricoles, les tâches ménagères et toutes les activités considérées comme impures. Les plus chanceux, généralement ceux qui travaillaient en tant que porteurs, pouvaient conserver une partie de leurs revenus et économisaient dans l'espoir de pouvoir acheter leur affranchissement ${ }^{7}$.

\section{6}

. H. Médard, M. L. Derat, T. Vernet et M. P. Ballarin, Traites et esclavages en Afrique orientale et dans l'océan Indien, Paris, Karthala, coll. «Esclavages», 2013 ; J. Armstrong, "Madagascar and the Slave Trade in the Seventeenth Century », Omaly sy Anio, vol. 17-19, 1984, p. 211-233; G. Campbell, «Madagascar and the Slave Trade, 1810-1895 », The Journal of African History, vol. 22, $\mathrm{n}^{\circ} 2$ 2, 1981, p. 203-227 ; L. Mosca, «La schiavitù e la sua abolizione in Madagascar », Africa. Rivista trimestrale di studi e documentazione dell'Istituto italiano per l'Africa e l'Oriente, vol. 52, n ${ }^{\circ} 4,1997$, p. 497-535; P. M. Larson, History and Memory in the Age of Enslavement: Becoming Merina in Highland Madagascar, 1770-1822, Portsmouth, Heinemann, 2000.

7

. Pour une description du statut et conditions de vie des esclaves, voir par exemple: B. Domenichini-Ramiaramanana et J.-P. Domenichini, «Regards croisés sur les grands sycomores, ou l'armée noire des anciens princes d'Imerina », Cheminements, Asie du Sud-Est et monde insulindien (ASEMI), vol. 11, n 1-4, 1980, p. 55-95 ; B. Domenichini-Ramiaramanana et J.-P. Domenichini, « Aspects de l'esclavage sous la monarchie merina d'après les textes législatifs et règlementaires », Omaly sy Anio, vol. 15, 1982, p. 53-98; G. Campbell, «Slavery and Fanompoana: The Structure of Forced Labour in Imerina (Madagascar), 1790-1861», Journal of African History, vol. 29, n², 1988, p. 463-486; 
Historiens et anthropologues ont reconstruit les trajectoires d'émancipation et de réassujettissement des esclaves et de leurs descendants après l'abolition, selon qu'ils étaient restés au service de leurs anciens maîtres, étaient rentrés dans leur village d'origine ou avaient occupé de nouvelles terres ${ }^{8}$. Ces études ont également analysé les discriminations auxquelles les descendants d'esclaves sont exposés actuellement dans de nombreuses régions de Madagascar. Sur les Hauts Plateaux, les descendants d'esclaves (andevo) sont souvent considérés comme impurs, sales et inférieurs ${ }^{9}$. Les mariages entre eux et les familles d'origine libre sont rares, même quand ils ont été reconnus comme propriétaires fonciers, ont eu accès à de nouvelles formes d'emploi ou sont parvenus à s'enrichir ${ }^{10}$. Les descendants d'esclaves constituent la couche la plus pauvre de la population dans plusieurs zones rurales (où, parfois, ils travaillent toujours comme métayers pour les descendants des anciens propriétaires), et dans certaines zones urbaines. Dans la ville d'Antananarivo ${ }^{11}$, ils occupent souvent les quartiers périphériques les plus défavorisés, situés en contrebas des collines où se trouvent le palais des anciens souverains merina et les quartiers de l'ancienne noblesse. Les descendants d'esclaves ont tenté de dissimuler leur origine et de s'approprier des attributs qui caractérisaient la condition de personne libre avant l'abolition, comme la construction des tombeaux de famille et la création d'une généalogie omettant la référence à un passé servile ${ }^{12}$. Dans plusieurs cas, ces

F. Ramiandrasoa, «Un aperçu sur la vie quotidienne des esclaves en Imerina au XIX ${ }^{\mathrm{e}}$ siècle », in I. Rakoto (dir.), L'esclavage à Madagascar ..., op. cit., p. 85-93.

8

. Voir, par exemple, M. Bloch, «Modes of Production and Slavery in Madagascar : Two Case Studies », in J.- L. Watson (dir.), Asian and African Systems of Slavery, Oxford, Basic Blackwell, 1979, p. 100-134 ; I. Rakoto et S. Urfer, Esclavage et libération à Madagascar, Paris, Karthala, 2014.

9

. S. J. T. M. Evers, «Expropriated from the Hereafter : The Fate of the Landless in the Southern Highlands of Madagascar », The Journal of Peasant Studies, vol. 33, n 3, 2006, p. 413-444 ; D. Graeber, Lost People. Magic and the Legacy of Slavery in Madagascar, Bloomington, Indiana University Press, 2007 ; D. Regnier, "Why Not Marry Them? History, Essentialism and the Condition of Slave Descendants Among the Southern Betsileo (Madagascar) », thèse de doctorat en anthropologie, London School of Economics and Political Science, 2012; M. Razafiarivony, «Les descendants des anciens esclaves importés d'Afrique à Madagascar : tradition et réalités », Journal of Asian and African Studies, $\mathrm{n}^{\circ} 70,2005$, p. 63-80.

10

. D. Regnier, "Clean People, Unclean People : the Essentialisation of "Slaves" among the Southern Betsileo of Madagascar», Social Anthropology, vol. 23, n² 2, p. 152-168.

11

. C. Fournet-Guerin, Vivre à Tananarive. Géographie du changement dans la capitale malgache, Paris, Karthala, coll. «Hommes et sociétés », 2007 ; C. Fournet-Guerin, «La géographie invisible de la ville. L'inscription des castes dans l'espace urbain à Tananarive (Madagascar) », in R. Sechet, I. Garat et D. Zeneide (dir.), Espaces en transactions, Presses universitaires de Rennes, 2008, p. 293-304 ; D. Nativel, Maisons royales, Demeures des Grands. L'inscription de la réussite sociale dans l'espace urbain de Tananarive au XIX ${ }^{e}$ siècle, Paris, Karthala, coll. « Hommes et sociétés », 2005 ; J.-M. Wachsberger, « Les quartiers pauvres à Antananarivo », Autrepart, vol. 51, n 3, 2009, p. 117-137; F. Roubaud, Ethnies et castes dans l'agglomération d'Antananarivo : facteur de divisions ou richesse culturelle?, Madagascar, Madio, Étude n 9733/E, 1997.

12

. Voir S. J. T. M. Evers, «The Construction of History and Culture in the Southern Highlands : Tombs, Slaves and Ancestors », in K. Middleton (dir.), Ancestors, Power and History in Madagascar, Leyde, Brill, 1999, p. 257-282 ; L. N. Razafindralambo, «Esclavage et inégalités, entre constructions 
stratégies n'ont pas abouti aux résultats espérés : le fait de ne pouvoir prouver que les caveaux avaient été construits avant l'abolition est souvent considéré comme une preuve d'ascendance servile.

Là où il est impossible de reconstruire les généalogies de chacun ou de connaître l'ancienneté des tombeaux de famille (en particulier dans les zones urbaines), d'autres critères sont utilisés pour déterminer si quelqu'un est ou non d'origine servile. Certains de mes interlocuteurs, d'origine noble et habitant les hauts quartiers d'Antananarivo, liaient la descendance servile aux caractéristiques phénotypiques : notamment un teint plus foncé ou les cheveux crépus. La dichotomie «Blanc» (fotsy) et «Noir » (mainty) s'est souvent imposée sur la distinction entre personnes d'origine libre (ou noble) et descendants d'esclaves, conduisant à une racialisation des catégories de différenciation sociale $^{13}$. Le terme mainty, qui, à l'origine, désignait un groupe de serviteurs royaux possédant un certain nombre de privilèges importants, est devenu au fil du temps un synonyme d'andevo ${ }^{14}$. Dans d'autres cas, mes interlocuteurs faisaient du lieu de résidence un critère caractéristique de ce statut : certains attribuaient les termes andevo ou mainty à tous ceux vivant dans les bas quartiers, bien que ceux-ci ne soient pas seulement peuplés de descendants d'esclaves, mais aussi de migrants de différentes régions de l'île n'ayant pas nécessairement une origine servile. Enfin, la pauvreté ou certains comportements (comme parler trop fort ou manger avec voracité) étaient souvent considérés comme des signes distinctifs. L'absence de débat public autour de la question de l'esclavage a rarement permis à ces critères d'être démentis publiquement et a contribué à étendre la catégorie d'andevo aux sujets positionnés en bas de la hiérarchie sociale. Dans un contexte où il est difficile de savoir «qui est qui », toute personne considérée comme un andevo devient un andevo et risque de ne pouvoir se marier avec les membres d'une famille d'origine libre ou noble.

\section{La politisation de l'ascendance servile}

Jean-Roland Randriamaro ${ }^{15}$ a consacré de nombreux travaux au rôle central des descendants d'esclaves dans les événements politiques de l'île après la seconde guerre mondiale. Il l'a montré que tant le Parti des déshérités de Madagascar (PADESM) que le

sociales et différences “naturelles" », in I. Rakoto et S. Urfer, Esclavage et libération ..., op. cit., p. 95-108 ; D. Somda, «De la pierre à la croix. Interprétation d'un paysage commémoratif (Anôsy, sud-est de Madagascar) », Études rurales, vol. 194, 2014, p. 79-102.

13

. L. N. Razafindralambo, «Esclavage et inégalités... », art. cité ; L. N. Razafindralambo, «Inégalité, exclusion... », art. cité.

14

. J. Ramamonjisoa, «"Blancs" et "Noirs". Les dimensions de l'inégalité sociale », Cahiers des sciences sociales, vol. 1, 1984, p. 39-75 ; B. Ramanantsoa Ramarcel, «Mainty = Andevo, un amalgame statutaire de l'Imerina », in I. Rakoto (dir), L'esclavage à Madagascar..., op. cit., p. 147-160.

15

. J.-R. Randriamaro, «L'émergence politique des mainty et andevo au $\mathrm{XX}^{\mathrm{e}}$ siècle », in I. Rakoto (dir), L'esclavage à Madagascar ..., op. cit., p. 357-381 ; J.-R. Randriamaro, PADESM et luttes politiques à Madagascar. De la deuxième guerre mondiale à la naissance du PSD, Paris, Karthala, 1997 ; J.R. Randriamaro, "Identité et métissages culturels chez les descendants d'esclaves de Tananarive ", in F. Rajaonah (dir.), Cultures citadines dans l'océan Indien occidental (XVIII-XXIe siècles), Paris, Karthala, 2011, p. 243-258. 
Parti social-démocrate (PSD, dirigé par Philibert Tsiranana, le premier président de Madagascar indépendant) visaient explicitement à devenir les défenseurs des intérêts des mainty et des andevo ${ }^{16}$. Tsiranana (président de la République de 1959 à 1972) a établi un régime explicitement pro-Français renforçant la perception selon laquelle la prétendue « indépendance », obtenue en 1960, n'était qu'un miroir aux alouettes. Les fortes relations entre l'ex-puissance coloniale et le gouvernement Tsiranana ont aussi renforcé l'idée, parmi de nombreux merina d'origine noble, que mainty, andevo et «côtiers » (le terme souvent utilisé pour désigner tous les groupes qui ne sont pas originaires des Hauts Plateaux) étaient alliés à la France afin de se prémunir d'une éventuelle réaffirmation de l'hégémonie merina sur l'île. Toutefois, le gouvernement de Tsiranana n'était pas en mesure de résoudre les problèmes de marginalisation sociale auxquels les descendants d'esclaves, en particulier dans la capitale, étaient exposés. Cela explique, entre autres, le rôle joué par les jeunes des bas quartiers dans la chute de la Première République.

En effet, en 1972, des groupes de jeunes descendants d'esclaves des bas quartiers d'Antananarivo et de jeunes étudiants militant en faveur de la réforme du système scolaire et universitaire ainsi que pour la malgachisation de l'enseignement, ont formé un mouvement marxiste et anti-impérialiste qui a contribué à la défaite du régime de Tsiranana ${ }^{17}$. Ces groupes ont composé la section la plus radicale du nouveau parti, le MFM (Mpitolona ho amin'ny Fanjakan'ny Madinika, Militants pour le pouvoir du prolétariat), dont le symbole (un homme noir portant un bandeau rouge et levant le poing) faisait explicitement allusion à la révolte des esclaves. "Zwam » était le nom que l'on donnait à ces bandes de jeunes chômeurs, peu scolarisés, qui vivaient dans les quartiers pauvres de la capitale et aimaient la boxe, le rugby et les westerns ${ }^{18}$. Plusieurs significations ont été attachées à cet acronyme. Pour certains, Zwam signifie Zatovo Western Andevo Malagasy (Jeunesse des esclaves western de Madagascar, western étant une référence à l'habitude de ces jeunes de porter des chapeaux de cow-boy). Pour

\section{6}

. Le cas de Madagascar n'est pas exceptionnel, si l'on considère d'autres contextes africains où des partis d'inspiration socialiste ont inclus la question des descendants d'esclaves dans leur agenda politique. Voir par exemple, B. Lecocq, «The Bellah Question : Slave Emancipation, Race, and Social Categories in Late Twentieth-Century Northern Mali », Revue Canadienne des Études Africaines, vol. 39, $\mathrm{n}^{\circ}$ 1, 2005, p. 42-68; Z. O. Ahmed Salem, «Bare-Foot Activists: Transformations in the Haratine Movement in Mauritania », in S. Ellis et I. van Kessel (dir.), Movers and Shakers. Social Movements in Africa, Leyde/Boston, Brill, 2009, p. 156-177 ; J.- F. Bayart, «Les Chemins de traverse de l'hégémonie coloniale en Afrique de l'Ouest francophone. Anciens esclaves, anciens combattants, nouveaux musulmans », Politique africaine, $\mathrm{n}^{\circ}$ 105, 2007, p. 201-240.

17

. Pour une description détaillée des événements de 1972, voir G. Althabe, «Les luttes sociales à Tananarive en $1972 »$, Cahiers d'Études africaines, vol. 20, n 80, 1980, p. 407-447 ; G. Althabe, « Strikes, Urban Mass Action and Political Change : Tananarive $1972 »$, in P. C. W. Gutkind, R. Cohen et J. Copans (dir.), African Labor History, Londres, Sage Publications, 1978, p. 205-243 ; F. Blum, « Madagascar 1972 : l'autre indépendance. Une révolution contre les accords de coopération », Le Mouvement social, $\mathrm{n}^{\circ} 236$, 2011, p. 61-87.

18

. Sur les Zoam, voir aussi J.-R. Randriamaro, «Aux origines des Zwam: les jeunes de bas quartiers de Tananarive, de l'entre-deux-guerres jusqu'à $1972 »$, in D. Nativel et F. Rajaonah (dir.), Madagascar revisitée, Paris, Karthala, 2009, p. 463-478 ; J.-R. Randriamaro, «Identité et métissages culturels... », art. cité. 
d'autres, qui considèrent le terme andevo comme une insulte, Zwam était l'acronyme de Zatovo Western Amerikanina Malagasy, Zatovo Western Amical Malagasy ou Zatovo Western Artistes Malagasy (Jeunesse des Américains/Amis/Artistes Western de Madagascar). Lorsque ces groupes ont trouvé un espace d'action dans la lutte politique marxiste, le mouvement a été rebaptisé Zoam (Zatovo Orin'Asa Malagasy, Jeunes chômeurs malgaches).

Devenus célèbres pour leurs affrontements violents avec les forces de l'ordre de Tsiranana, qui avaient tiré sur la foule pendant les manifestations des 13 et 15 mai 1972, les jeunes des bas quartiers devinrent une des composantes fondamentales (avec les étudiants, les enseignants et les travailleurs) du Comité commun de lutte (KIM, Komity Iraisan'ny Mpitolona). Cet organisme disait représenter les différents groupes contestataires et avait organisé de grandes assemblées publiques, des manifestations et des séminaires de discussion pendant les derniers mois de la présidence de Tsiranana. Les colonnes du journal Andry (Pilier) devinrent leur principal canal d'expression. Comme l'écrit Gérard Althabe :

«Les militants Zoam occupent dans la ville une position marginale, ils sont jeunes, ils ont été rejetés très tôt de l'école, ils sont exclus du salariat bureaucratique ; dans l'univers urbain où les niveaux hiérarchiques sont particulièrement différenciés, ils occupent la position la plus basse. Leur adhésion passionnée à l'utopie égalitaire et à l'« État des petits », célébrés dans des poèmes et des chants, exaltés dans des assemblées de plus de cinq cents participants au foyer culturel où siègent les dirigeants du mouvement, est le reflet de la place qui leur est faite dans la ville : au plus bas dans l'échelle de la hiérarchie, ils adoptent d'autant plus intensément l'utopie qui la nie ${ }^{19}{ }$.

La polysémie de l'acronyme Zwam renvoyait à l'embarras que suscitait l'auto-attribution d'un terme péjoratif comme andevo. Ce terme était rejeté soit par certains descendants d'esclaves, soit par les immigrés (ou les enfants d'immigrés) d'origine libre des bas quartiers, qui ne voulaient pas partager avec les descendants d'esclaves le stigmate de l'origine servile. En ce sens, la transformation de Zwam en Zoam n'a pas seulement signifié le passage de l'imaginaire des westerns à celui du marxisme, mais elle a également permis de rassembler des jeunes d'origines différentes autour de la dénomination de «chômeurs », une catégorie moins stigmatisante que celle d'esclaves. Toutefois Zoam n'a pas renoncé à mobiliser la question de l'esclavage. Au contraire, le mouvement s'est servi de ce thème comme d'une arme politique efficace. Pendant la révolte de 1972, les accords de coopération avec la France ont été dénoncés et rebaptisés en tant qu' «accords d'esclavage » : la relation de dépendance économique avec l'ancien pouvoir colonial représentait un terreau fertile pour y appliquer la métaphore esclavagiste $^{20}$, liant par extension Zoam aux anciens mouvements de lutte anticoloniale. Chansons, poèmes et vocables explicitement liés au thème de l'esclavage ont été mobilisés pour contester l'hégémonie des Français sur l'île, mais aussi pour restaurer la

19

20

. G. Althabe, «Les luttes sociales à Tananarive... », art. cité, p. 447.

. C. Rafidinarivo Rakotolahy, «Le réfèrent de l'esclavage dans les représentations transactionnelles marchandes à Madagascar », Journal des africanistes, vol. 70, n 1, 2000, p. 123-144. 
dignité des groupes qui avaient été stigmatisés jusqu'alors par l'élite merina. Durant cette période, les termes qui désignaient les esclaves ou leurs descendants (comme andevo, mais aussi zazamanga «enfant bleu »: une expression infantilisante qui indiquait les esclaves d'origine africaine) ont été réappropriés, en signe de provocation, par les militants les plus radicaux ${ }^{21}$.

La métaphore de l'esclavage rassemblait à la fois ceux qui soutenaient un programme nationaliste anti-français et ceux qui, bien que critiques envers l'hégémonie française, luttaient non seulement contre les accords de coopération franco-malgaches ou en faveur de la malgachisation de l'enseignement, mais aussi contre les discriminations locales fondées sur la hiérarchisation entre groupes statutaires ou entre «Blancs » et « Noirs ». Pour de nombreux militants, le marxisme offrait un cadre théorique permettant de remettre en question les inégalités entre groupes statutaires et de les transcender en faveur d'une lutte de classe liée à une campagne plus large contre l'impérialisme occidental. L'image de Che Guevara, mai 1968 à Paris, le Livre rouge de Mao et les mouvements pour les droits civiques des Noirs aux États-Unis devinrent, en 1972, des symboles attractifs pour beaucoup de jeunes malgaches des bas quartiers.

Et pourtant, Zoam a disparu de la scène politique peu après y être apparu. Devenu président de la République en 1975, Didier Ratsiraka ${ }^{22}$ s'est approprié la rhétorique marxiste pour consolider son pouvoir. Il a rassemblé certains groupes de jeunes chômeurs des bas quartiers au sein des TTS (Tanora Tonga Saina, Jeunes conscientisés) et s'en est servi pour contenir, par la violence, toute opposition ${ }^{23}$. Auprès des habitants d'Antananarivo (surtout ceux résidant dans la haute ville), les vols et les menaces des TTS ont contribué à renforcer le stéréotype confondant les jeunes des bas quartiers avec

21

. Comme Jean-Roland Randriamaro l'a montré, le journal officiel de MFM et de Zoam (Andry) publia plusieurs poèmes qui liaient la figure de l'esclave rebelle à celle du guérillero marxiste. Un poème intitulé Zazamanga, notamment, se concluait par ces mots : «Moi l'andevolahy/Le Noir aux cheveux crépus/Moi la raclure/Chien errant, esclave sans valeur/Moi le zazamanga/Chien de voyou qui va s'émanciper/Moi l'esclave des ancêtres/Résolu de vaincre, défiant, les dents serrées/Moi le guerrier/Le guérillero, j'ouvrirai le front/Tremble ô monde ! Moi l'andevolahi, les dominés s'insurgent », cité in J.R. Randriamaro, «L'émergence politique des mainty... », art. cité. Les références aux militants de Zoam (appelés jomaca) reviennent aussi dans une des chansons les plus connues à Madagascar : Lendrema. La chanson, composée dans les années 1970 par un groupe de jeunes musiciens connus sous le nom de Mahaleo (et devenu, par la suite, le plus célèbre groupe musical de l'île), décrit les conditions de vie des jeunes des bas quartiers avec une référence explicite à l'esclavage : "Le fils de Lendrema/L'orphelin de Lendrema/Il est né dans le marché/Il est fils de l'andevo (esclave) qu'on ne veut pas aimer/Il est l'enfant indiscipliné qui ne vaut pas une signature/ll est le fils du peuple/Même s'il est le fils de jomaca/Pourquoi riez-vous? » (traduction de l'auteur).

22

. Originaire de la province de Tamatave, Didier Ratsiraka est devenu président de la République pour la première fois en 1975. En 1993, il a perdu les élections contre Albert Zafy. En 1997, il est revenu à la tête de l'État, où il est resté jusqu'en 2002, date à laquelle Marc Ravalomanana a remporté les élections. 23

. Sur le régime de Ratsiraka et sa chute, voir notamment B. A. Gow, « Admiral Didier Ratsiraka and the Malagasy Socialist Revolution », The Journal of Modern African Studies, vol. 35, $\mathrm{n}^{\circ}$ 3, 1997, p. 409-439 ; F. Raison-Jourde, «Une transition achevée ou amorcée ?», Politique africaine, $\mathrm{n}^{\circ}$ 52, 1993, p. 6-18 ; F. Raison-Jourde et J.-P. Raison (dir.), «Madagascar, les urnes et la rue », Politique africaine, $n^{\circ}$ 86, 2002 ; S. Randrianja, "Be Not Afraid, Only Believe": Madagascar 2002 », African Affairs, vol. 102, n$^{\circ} 407,2003$, p. 309-329. 
des délinquants prêts à se vendre au régime pour une poignée de riz. Le MFM, qui avait intégré les Zoam en tant que cadres du parti, perdit les élections de 1977 pour les collectivités décentralisées. Le président de MFM attribua ce fiasco à la présence de trop de candidats noirs pour un parti qui aspirait peu à peu à représenter toute la société et pas seulement une de ses fractions ${ }^{24}$. Le terme jomaca, désignant les militants de Zoam, perdit la valeur politique qu'il avait acquise dans les années soixante-dix pour devenir synonyme de jeunes pauvres, violents et potentiellement criminels.

La politique pro-française du PSD et le régime autoritaire de Ratsiraka ont non seulement largement discrédité l'activisme politique des descendants d'esclaves, mais ils ont aussi contribué à renforcer l'idée, déjà soutenue par certaines sections merina d'origine noble ou libre, que les descendants d'esclaves ou les « Noirs » n'étaient pas aptes à gouverner, étant par «nature » incapables, violents ou prêts à se vendre aux «étrangers : Français, Indiens, Chinois, ou Américains ». Pour certains de mes interlocuteurs merina et betsileo d'origine noble, de l'indépendance jusqu'à la chute de Ratsiraka en 2002, le pays a été gouverné par des «esclaves » (allusion à Richard Ratsimandrava, président d'origine servile assassiné quelques jours après sa nomination en 1975) ou par des «côtiers » (comme Tsiranana ou Ratsiraka). Ce sont, selon eux, deux catégories qui nourriraient encore une profonde rancœur envers les nobles merina et dont les ancêtres auraient profité du soutien français soit pour s'affranchir (dans le cas des esclaves), soit pour se débarrasser du contrôle que l'empire merina avait étendu sur leurs régions d'origine au cours du XIX $\mathrm{e}^{\mathrm{e}}$ siècle (dans le cas des « côtiers »). Ils élaboraient une vision nostalgique de l'histoire merina précoloniale qui omettait toute référence aux violences commises par les anciens souverains et refusaient tout droit de gouverner aux mainty, qu'ils considéraient comme des «étrangers d'origine africaine ». En revanche, d'autres interlocuteurs rencontrés dans les bas quartiers de la capitale croyaient que, au-delà des origines des présidents, les véritables détenteurs du pouvoir à Madagascar étaient l'élite économique merina d'origine libre et noble, qui aurait préféré placer des marionnettes à la tête du gouvernement afin de contrôler le pays sans toutefois être exposée directement. Comme Jean-Pierre Raison $^{25}$ 1'a indiqué dans le cas des élections de 1993 qui ont conduit à la première défaite de Ratsiraka, «la géographie électorale malgache est plus finement maillée » qu'une simple opposition entre merina et «côtiers » (ou entre «Blancs » et « Noirs »), et elle n'a pas nécessairement suivi des logiques de « caste » ou d' « ethnie ». Toutefois, ces interprétations simplificatrices de l'histoire politique postcoloniale structurent le domaine de la discussion politique dans un contexte où il est difficile d'avoir des informations précises sur ce qui se passe (ou s'est passé) dans les coulisses du pouvoir. En interprétant l'histoire politique malgache par l'opposition entre groupes statutaires, entre «Blancs» et «Noirs » ou entre Merina et «côtiers », ces discours réaffirment la pertinence de ces catégories de différenciation sociale et expliquent en partie pourquoi elles n'ont pas disparu avec l'abolition de l'esclavage ou l'indépendance. Chargées de nouvelles valeurs politiques, ces catégories sont devenues des grilles de lecture du champ politique du pays. Et pourtant, si l'opposition entre Merina et

24

25

. J.-R. Randriamaro, «L'émergence politique des mainty... », art. cité, p. 375.

. J.-P. Raison, «Une esquisse de géographie électorale malgache : le premier tour des élections présidentielles (novembre 1992)», Politique africaine, $\mathrm{n}^{\circ}$ 52, 1993, p. 74. 
« côtiers » a été mobilisée publiquement - par exemple quand Ratsiraka a essayé en vain de l'instrumentaliser pour garantir le vote des «côtiers» en sa faveur pendant sa confrontation électorale avec le «Merina» Ravalomanana ${ }^{26}$ en 2002 -, la distinction entre groupes statutaires reste beaucoup plus implicite. Après la disparition de Zoam, la question des discriminations envers les descendants d'esclaves est sortie du débat public. Toutefois, le mouvement n'a pas disparu sans laisser de traces.

\section{La honte et la fierté des « esclaves »}

Plusieurs tendances ont cohabité au sein de Zoam : le mouvement a été à la fois un catalyseur des protestations juvéniles contre le régime Tsiranana, un héritier marxiste de la lutte anticoloniale, une plateforme de relance du discours nationaliste sous l'emblème de la malgachisation de l'enseignement et un espace hétérogène de politisation pour les étudiants et les groupes de jeunes des bas quartiers d'Antananarivo. Pour beaucoup d'entre eux, l'activité politique de Zoam a représenté un bon exemple de ce qu'Erving Goffman $^{27}$ a appelé le « retournement du stigmate », à savoir la tentative de redonner une dignité aux groupes stigmatisés (ici, pour leur origine servile) et de transformer cette stigmatisation en arme politique. L'analyse des trajectoires de vie et des discours des exmilitants est cruciale pour mieux comprendre les ambiguïtés et les contradictions que cette stratégie a engendrées, notamment la tension entre la volonté de remettre explicitement en question le stigmate lié à la descendance servile et le risque de reproduire des formes de stigmatisation en s'appuyant sur les mêmes catégories que l'on se propose d'éradiquer. Les entretiens que je présente donnent une idée des contradictions auxquelles l'activisme politique de Zoam est confronté et illustrent la pluralité des développements d'un discours politique tentant de lier la lutte des classes aux combats contre les discriminations entre groupes statutaires. Cette approche permet de comprendre les raisons de la disparition de Zoam ainsi que les différents héritages de ce mouvement.

Si certains militants Zoam sont rentrés dans les TTS, d'autres ont maintenu une attitude critique envers le régime de Ratsiraka et se sont retirés de la scène publique. Certains ont traversé toutes ces étapes. L'un d'eux, un homme originaire du bas quartier d'Isotry, m'a raconté comment, après avoir été membre de Zoam (il avait 16 ans en 1972), il a été

\section{6}

. Homme d'affaires d'origine merina et fondateur de l'une des plus importantes entreprises de produits laitiers de Madagascar, Marc Ravalomanana devient président de la République en 2002, après des élections contestées qui ont conduit à la défaite de Ratsiraka (voir S. Randrianja, " "Be Not Afraid, Only Believe"...», art. cité ; J. Cole, "Malagasy and Western Conceptions of Memory: Implications for Postcolonial Politics and the Study of Memory », Ethos, vol. 34, n 2, 2006, p. 211-243). Ravalomanana reste au pouvoir jusqu'en 2009, date à laquelle un coup d'État organisé par Andry Rajoelina, un autre jeune entrepreneur, initie la crise politique et la période de «transition», qui se conclut officiellement avec l'élection en 2014 d'Hery Rajaonarimampianina. Pour une analyse de l'affrontement entre Marc Ravalomanana et Andry Rajoelina ainsi que du rôle des bas quartiers dans la controverse, voir D. Galibert, "Mobilisation populaire et répression à Madagascar. Les transgressions de la cité cultuelle », Politique africaine, $\mathrm{n}^{\circ} 113,2009$, p. 139-151.

27 commun », 1975.

. E. Goffman, Stigmate. Les usages sociaux des handicaps, Paris, Minuit, coll. «Le sens 
convaincu par un ami de rejoindre les TTS. Celui-ci m'a parlé avec honte des abus, des viols, des violences infligées aux adversaires potentiels du régime et des vols qu'il avait commis pendant cette période. Après un certain temps, il a décidé de quitter les TTS et a changé de camp politique. Il a même participé en 1984, avec des membres de «Kung$\mathrm{Fu} »$ (un groupe paramilitaire d'autodéfense formé par de jeunes pratiquants d'arts martiaux, critiques envers le régime ${ }^{28}$, à l'attaque du siège des TTS. L'assaut a fait des dizaines de morts et a été suivi par les représailles du gouvernement qui ont conduit à la mort et à l'arrestation de nombreux membres de Kung-Fu. Après ces événements, cet homme s'est retiré de la vie publique et gère désormais un petit commerce de vente au détail. Il a décrit avec regret son passage de Zoam à TTS, expliquant comment le rêve d'une société égalitaire né dans les années soixante-dix a ensuite été manipulé et corrompu par l'arrivée au pouvoir de Ratsiraka :

« Nous avons lutté contre la stigmatisation de la descendance servile et contre les inégalités de classe. À la fin, nous avons fini par commettre des crimes qui ont donné raison à ceux qui disaient que les jeunes des bas quartiers étaient des voyous. Quoi qu'il en soit, nous étions pauvres et nous sommes restés pauvres. Rien n'a changé ${ }^{29} »$.

Pour lui, la lutte politique en tant que « Noirs » ou « descendants d'esclaves » n'a fait que renforcer la discrimination envers les bas quartiers. Ses idées sont partagées par la plupart des ex-militants de Zoam que j'ai rencontrés. Beaucoup d'entre eux exprimaient leurs remords d'avoir contribué au renversement du régime de Tsiranana et leur déception de ne pas avoir réussi à replacer au centre du débat la question de la stigmatisation à laquelle ils ont continué d'être confrontés dans leur quotidien. "Il a été beaucoup plus facile de se débarrasser de Tsiranana que de changer les idées de ceux qui méprisent les habitants de bas quartiers ${ }^{30} »$, disait un autre ex-militant de Zoam. Né à Isotry et fils d'un migrant betsileo d'origine libre, ce dernier n'a pas pu épouser la fille qu'il aimait car il n'est jamais parvenu à convaincre le père de celle-ci (un Merina d'origine libre vivant dans les quartiers de la haute ville) qu'il n'était pas un descendant d'esclaves : "Il savait que j'étais un Zoam et, pour lui, être Zoam était synonyme d'andevo et de TTS ».

$\mathrm{Au}$ contraire, d'autres anciens militants Zoam rencontrés en 2014 se rappellent le mouvement de 1972 avec fierté, non seulement parce qu'il a contribué à la chute de Tsiranana et à une renégociation temporaire du rôle de la France dans les affaires intérieures de Madagascar, mais aussi parce qu'il a donné une dignité à ceux qui étaient considérés comme «Noirs» ou descendants d'esclaves. Comme me l'a expliqué un homme originaire du quartier d'Andavamamba, passé « des bagarres de quartier à la lutte anti-impérialiste, des films westerns à Che Guevara ${ }^{31} »$ grâce à Zoam, le marxisme a enseigné que «c'étaient les patrons qui devaient avoir honte, pas les esclaves. Zoam nous

28

. Voir «Les Kung-Fu» dans F. Raison-Jourde, «Le pouvoir en double », Politique africaine, $\mathrm{n}^{\circ} 86,2002$, p. 68-69.

29

30

. Entretien avec M. Arivo, Antananarivo, 4 juin 2014.

. Entretien avec M. Héry, Antananarivo, 6 juillet 2014.

31

. Entretien avec M. Charles, Antananarivo, 15 juin 2014. 
a appris à rejeter le complexe d'infériorité envers les soi-disant "nobles". Il ne faut pas se cacher, il faut lutter ». Cette déclaration montre comment le marxisme a été mobilisé pour interpréter et critiquer les formes locales de marginalisation sociale, en produisant un contre-discours, certes minoritaire mais non moins important, pour contester l'idée selon laquelle la seule façon pour les descendants d'esclaves d'éviter la stigmatisation était de cacher leur origine : «En tant que mainty, nous étions discriminés. Et donc, en tant que mainty nous avons répondu. Nous avons montré comment c'est dur la tête des esclaves !». Cet homme, qui a renoncé à ses activités politiques après la prise du pouvoir par Ratsiraka et a travaillé comme maçon toute sa vie sans jamais quitter le quartier où il est né, a commenté avec satisfaction le fait que les habitants de la haute ville avaient peur de s'aventurer dans les bas quartiers. "Ils ont peur de nous, maintenant », disait-il avec orgueil. Pour lui, le mouvement Zoam, sans avoir pleinement atteint ses objectifs, avait au moins laissé, dans la mémoire de la capitale, la perception que les bas quartiers pouvaient exercer une certaine influence sur la politique nationale et que la «tradition », si elle était une manière de légitimer un pouvoir basé sur les divisions de « caste » ou de classe, devrait être proscrite. Bien qu'il fût critique envers le régime Ratsiraka, il considérait cette période comme plus propice que la situation actuelle : «Le riz était moins cher et le gouvernement faisait beaucoup de choses pour les bas quartiers. Ceux qui sont arrivés au pouvoir après ont fait seulement beaucoup de promesses. Il n'y a plus de place pour les mainty pauvres dans la politique nationale ». Pour lui, le dogme néolibéral qui a monopolisé le débat public à partir de la fin des années 1980 n'a pas amené la richesse promise et a réduit les possibilités d'émergence politique des bas quartiers : «Aujourd'hui, la devise est "chacun pour soi" et les seuls à profiter vraiment de ça sont les riches entrepreneurs qui achètent leurs votes ici, dans les bas quartiers, pendant les campagnes électorales ».

L'activisme politique a remodelé la perception que certains jeunes appartenant à la couche la plus discriminée de la société malgache avaient d'eux-mêmes. Il a également contribué à former l'idée d'une appartenance à une communauté qui transcendait potentiellement les divisions entre groupes statutaires. En fait, le mouvement n'était pas composé uniquement de jeunes descendants d'esclaves, mais aussi d'une jeunesse paupérisée d'origine libre et d'étudiants d'origine noble, luttant côte à côte contre le régime de Tsiranana et sa subalternité vis-à-vis de la France. La présence d'un ennemi commun, l'adhésion à une idéologie égalitaire et la sensation d'appartenir à une génération qui peu à peu imposait son agenda politique au niveau national, ont été des facteurs qui ont permis à beaucoup de jeunes de surmonter les distinctions entre groupes statutaires.

En 2014, j'ai rencontré un de ces (ex-) étudiants à Ambositra ${ }^{32}$. L'homme était originaire d'une famille noble de Fianarantsoa et a étudié à l'université d'Antananarivo pendant la période d'activité de Zoam. Il a fréquenté avec assiduité les réunions de MFM et a eu l'occasion de rencontrer un grand nombre de militants des bas quartiers. Cette expérience l'a conduit à repenser de manière critique les discriminations envers les descendants d'esclaves, au point de déclarer que jamais il n'aurait empêché un de ses descendants d'épouser la personne de son choix. Pour lui, et d'autres descendants de nobles et de personnes libres sympathisants de la « cause des bas quartiers », les divisions de « caste » 
devaient être explicitement combattues et avec la même vigueur que celle employée pour lutter contre l'inégalité économique entre les riches et les pauvres (une division qui ne chevauchait pas nécessairement celle entre «Blancs» et « Noirs »). Il constatait avec amertume que les questions soulevées par Zoam avaient disparu du débat politique national et qu'aucun parti n'était désormais capable de répondre aux besoins des bas quartiers. Même le MFM avait «trahi les plus pauvres", après avoir embrassé le néolibéralisme dans les années 1990. Pour lui, la tentative visant à forger une conscience de classe qui unirait ces pauvres n'avait abouti à aucun résultat probant. Après avoir compris que Ratsiraka était un dictateur, les gens des bas quartiers ont commencé à voter pour «les Ravalomanana, les Rajoelina...", tous des riches entrepreneurs merina «blancs » qui, selon lui, ont instrumentalisé les bas quartiers pour accéder au pouvoir, puis ont oublié leurs promesses. Il ajoutait que, grâce à Zoam, les jeunes des bas quartiers avaient été les principaux protagonistes de la scène politique pendant une brève période, mais que, depuis Ratsiraka, ils étaient redevenus les marionnettes qu'ils étaient auparavant : « Ils votent pour celui qui les paie, maintenant».

Étant d'origine noble, son expérience de militant créa un conflit familial. Son père et d'autres membres de sa famille déploraient qu'il ait été impliqué dans les «affaires des andevo ». Ils tentèrent, en vain, de lui interdire de poursuivre son activité politique. À l'heure actuelle, des pans entiers de sa famille critiquent encore son combat autour de la nécessité d'« abolir » les interdictions matrimoniales. Il a toutefois transmis ses idées à ses enfants. Ces derniers ne s'intéressaient pas à «qui était qui ». Ils voyaient d'un mauvais œil les discriminations matrimoniales et considéraient ces tentatives comme implicitement liées à l'agenda politique de nobles qui s'attachaient aux questions de statut pour légitimer certaines revendications politiques, ou qui, parce que désormais appauvris, n'avaient rien d'autre à défendre. Leurs opinions étaient partagées par la plupart des jeunes (d'origine libre ou servile, Merina et «côtiers ») rencontrés dans les bas quartiers d'Antananarivo. Ces jeunes n'étaient pas des activistes politiques et connaissaient les événements de 1972 essentiellement à travers les témoignages de leurs parents. Plus d'une fois ils ont souligné que «continuer à parler de andriana, hova et andevo » était, dans une certaine mesure, une façon de ne pas se concentrer sur les formes contemporaines d'exploitation auxquelles les segments les plus pauvres de la population sont soumises, indépendamment de leur statut. Leur position ne visait pas à échapper à la question - jugée importante, surtout sur le plan matrimonial et parce que les plus pauvres étaient souvent des descendants d'esclaves vivant dans les bas quartiers de la capitale mais souhaitait attirer l'attention sur les différences de classe afin de changer un ordre de discours fondé sur des catégories incapables, selon eux, de rendre compte des nouvelles formes de marginalisation sociale.

Si on compare leurs idées avec la position des ex-militants exposée plus haut, on peut comprendre la contradiction à laquelle l'activisme politique de Zoam est confronté. En effet, les discriminations entre groupes statutaires et les inégalités de classe ne se chevauchaient pas parfaitement, même à Antananarivo où il existe d'importantes intersections entre les deux. Ces dernières ont permis à Zoam d'utiliser le langage de classe pour décrire les inégalités entre groupes statutaires et, en même temps, la référence à l'esclavage pour dénoncer la marginalisation économique et social des bas quartiers. Cela n'a pas résolu la contradiction entre le combat plus général pour l'amélioration des conditions de vie du prolétariat urbain et la lutte, plus spécifique, pour briser la honte liée 
à l'ascendance servile, Pour certains des ex-militants Zoam, lutter en tant que « Noirs » ou «descendants d'esclaves » est la seule façon de dénoncer explicitement un «secret public » qui a imprégné la société en profondeur. Cacher la question derrière un discours de classe, selon eux, renforcera la honte qui pèse sur l'ascendance servile. Par conséquent, lutter pour l'amélioration des conditions de vie des bas quartiers, bien que cela reste fondamental, ne suffira pas à éradiquer la discrimination qui perdure aussi dans des contextes où descendants de personnes d'origine libre et descendants d'esclaves partagent les mêmes conditions de vie ${ }^{33}$. Mais leur position reste fortement minoritaire et ne parvient plus à se concrétiser dans un activisme politique explicite.

En effet, pour la plupart des ex-Zoam, conduire une lutte en tant que «descendants d'esclaves » ne peut qu'aboutir à de nouvelles formes de discrimination ou à des instrumentalisations politiques réaffirmant la pertinence des mêmes distinctions entre les groupes statutaires qu'on se propose d'éradiquer. Ils préfèrent reformuler la question en termes de classe pour aboutir à un front commun avec les couches sociales des bas quartiers qui n'ont pas - ou ne veulent pas s'attribuer - une ascendance servile. Mais ils remarquent aussi que le langage de classe, après avoir été instrumentalisé et délégitimé par la politique autoritaire de Ratsiraka, semble avoir perdu beaucoup de sa capacité d'attraction et qu'aucun parti n'est en mesure, à l'heure actuelle, de représenter les intérêts des bas quartiers. Chacun des ex-militants de Zoam que j'ai rencontrés a déclaré ne pas aller voter et ne plus avoir confiance dans l'ensemble des partis politiques ${ }^{34}$.

\begin{abstract}
À Madagascar, les subdivisions entre groupes statutaires ont continué à structurer, parfois de manière indirecte ou implicite, les champs politiques et sociaux locaux, et à fournir des points de référence susceptibles d'être connotés de nouvelles valeurs politiques. L'activisme politique des descendants d'esclaves a été à la fois une conséquence et une cause de ce processus et a généré des résultats contradictoires. Même si Zoam a réussi à rassembler un grand nombre de personnes d'origine différente dans un front de lutte commun, le mouvement a échoué à briser la honte qui pèse sur l'ascendance servile. Si pour certains membres de Zoam le marxisme avait redonné une dignité aux descendants d'esclaves, pour la plupart il est devenu une façon de dissimuler une question épineuse derrière un discours de classe qui n'a en rien réduit la fragmentation politique et sociale des bas quartiers. Les partis politiques malgaches, quant à eux, ont préféré éviter toute référence à la persistance de groupes statutaires pour ne pas perdre les portions de votants qui méprisaient l'ascendance servile et ont profité de la pauvreté des bas quartiers pour acheter des votes à bas prix pendant les campagnes électorales. Les gangs de jeunes chômeurs sont devenus utiles pour déstabiliser le gouvernement ou, au contraire, pour attaquer les opposants. Les événements de 1972 et les violences des TTS
\end{abstract}

\title{
33
}

34

. Voir notamment D. Regnier, « Clean People, Unclean People... », art. cité.

. Lors des élections communales du 31 juillet 2015, le taux d'abstention a atteint $70 \%$ à Antananarivo. République de Madagascar, commune d'Ambanitsena, «Annexe de la délibération $\mathrm{N}^{\circ} 001$ SADIR-TANA/15 du 12 août 2015 portant publication des résultats provisoires des élections Communales et Municipales du 31 juillet $2015 », \quad 11$ août 2015, <cenitmadagascar.mg/bdcommunale/pdf_commune/111302.pdf〉, consulté le 06 novembre 2015. 
ont servi de modèle pour toutes les tentatives, réussies ou avortées, de prise ou de conservation du pouvoir dans la capitale. Les actions violentes de ces gangs ont souvent renforcé les stigmates attachés aux bas quartiers.

Pourtant, Zoam a joué un rôle considérable pour ceux qui, sans avoir une origine servile, ont, malgré tout, soutenu le mouvement. Comme le cas du noble betsileo l'a montré, l'expérience de Zoam a contribué à modifier sensiblement leur attitude envers les divisions de «caste» et a permis l'émergence d'un contre-discours qui, bien que minoritaire, doit être pris en considération. Si l'analyse et la déconstruction du discours de ceux qui méprisent l'ascendance servile restent cruciales pour dénoncer les structures de pouvoir et les idéologies sur lesquelles les inégalités sociales s'articulent au niveau local, il faut comprendre aussi pourquoi beaucoup de personnes d'origine libre ou noble ne sont plus obsédées par le désir de savoir «qui est qui ». Pour beaucoup de jeunes ayant participé à la révolte de 1972, l'activisme politique a valorisé des liens politiques, sociaux et générationnels qui ont transcendé les divisions entre groupes statutaires et a fourni un contexte privilégié pour l'élaboration d'une critique de la stigmatisation de l'origine servile. Focaliser l'attention sur les représentations de ceux qui ont tenté de remettre en question cette stigmatisation, comme les militants ou les sympathisants du Zoam, permet non seulement de montrer le rôle de l'activisme politique dans les trajectoires de vie des habitants des bas quartiers, mais aussi de souligner comment il a contribué à remettre en question l'hégémonie des discours dominants. L'héritage de Zoam, même s'il ne se traduit plus dans des luttes politiques explicites, restitue une image moins statique ou homogène de la société et aide à comprendre comment le stigmate associé à la descendance servile a été non seulement reproduit, mais aussi renégocié et contesté au niveau local.

\author{
Marco Gardini \\ Université de Milano-Bicocca
}

\begin{abstract}
Political Activism of Slave Descendants in Antananarivo: The Legacies of Zoam

This article analyzes the legacies that a Malagasy Marxist movement, born in 1972 and composed by young people of servile origin, left in the political trajectories and in the discourses of former militants and of the inhabitants of the poor neighborhoods of Antananarivo. It considers the role this movement had in the renegotiation of the social position of slave descendants in the last decades, the life trajectories of activists after its disappearance and how the stigma attached to servile origin has been reproduced, criticized and charged with new political values.
\end{abstract}

